\title{
Aporte de las TIC a la gestión administrativa de centros educativos unidocentes del Circuito 03 de San Ramón en el distrito de Piedades Sur
}

Aldous Brenes Espinoza

Licenciado en Administración Educativa egresado del Programa de Administración Educativa, UNED, Costa Rica; aldousbe10@hotmail.com

Recibido: 09 de agosto del 2018

Corregido: 31 de agosto del 2018

Aceptado: 17 de setiembre del 2018

\section{Resumen}

El artículo es el resultado de una investigación cuyo objetivo fue conocer los aportes que las TIC generan a la gestión administrativa de centros educativos unidocentes. Los aportes se investigaron con profundidad en la tesis denominada "Los aportes de las tecnologías de información y comunicación, a la gestión administrativa de centros educativos unidocentes del Circuito 03 de San Ramón, distrito de Piedades Sur en el 2016 y 2017". Mediante una entrevista semiestructurada se recolectaron los datos y se manifiesta que el uso de las tecnologías de información y comunicación en el área de gestión administrativa es prácticamente nula, y la usabilidad de la misma se limita a la creación de documentación y comunicación. Contrario al resultado, se evidenció que a nivel pedagógico existe una utilización más efectiva del uso de las TIC; sin embargo, las capacitaciones en este ámbito son más frecuentes y hay todo un respaldo teórico y pedagógico creado por el Programa de Informática Educativa PRONIE-MEP-FOD.

Palabras clave: Tecnologías de información y comunicación, escuela pequeña, formación, Informática Educativa, gestión, innovación educacional.

\section{Abstract \\ Contribution of the TIC to the administrative management of unidocentes educational cen- ters in the 03 Circuit in San Ramón, Piedades Sur district}

This article is the result of an investigational that takes as a target to know the contributions that are generating the TIC to the administrative management of Unidocentes Educational Centers. These contributions were investigated by depth in the so called thesis "The contributions of the technologies of information and communication, to the Administrative management of Unidocentes Educational Center in the 03 Circuit in San Ramón, Piedades Sur district". By means of a semiestrctured interview there were gathered the information, where one shows the use of the technologies of information and communication in the field of administrative management is practically void, and the usability of the same one limits herself to the creation of papers and communication. The opposite to this result, it was demonstrated that at pedagogic level there is a more effective TIC use; nevertheless the trainings in this ambience are more frequent and there is the whole theoretical and pedagogic support created by the Program of Computer use in education PRONIE-MEP-FOD.

Key words: Technologies of information and communication, small school, formation, informatics education, management, innovation education. 


\section{INTRODUCCIÓN}

Los resultados aquí presentados se inscriben en el contexto de una investigación realizada durante 2016 y 2017, con el fin de conocer cuáles han sido los aportes de las TIC a la gestión administrativa de escuelas unidocentes seleccionadas. Para ello se estudió el uso y la frecuencia con que se empleaban las herramientas tecnológicas y su repercusión en la comunicación, toma de decisiones y planificación.

Las tecnologías de información y comunicación, conocidas con las siglas (TIC), son herramientas que permiten mejorar a nivel interno y externo los sistemas educativos; mediante su aplicación se genera y se procesa el conocimiento de forma dinámica, se promueve la democracia, la cultura. Además, hace de la comunicación un mecanismo de fácil acceso (Fundación Omar Dengo, 2006).

La inclusión de las tecnologías en la educación costarricense es una realidad que se viene evidenciando desde 1989, sobre todo mediante la creación de políticas educativas que han respaldado su aplicación. Con el fin de brindar una educación de mejor calidad, se crea el Programa Nacional de Informática Educativa, mediante un convenio entre el Ministerio de Educación Pública y la Fundación Omar Dengo (en adelante abreviado a PRONIE MEP-FOD). La creación del programa ha permitido el abastecimiento de recursos tecnológicos a más de 1200 escuelas unidocentes y multigrado en todo el territorio nacional. Esta dotación de recursos incluye computadora portátil para cada estudiante y profesor, proyector, equipo de sonido y un sistema de red de comunicación. El proyecto es financiado con fondos públicos y representa una oportunidad que acerca a las escuelas en busca de una educación de calidad.

El uso de los equipos tecnológicos es prácticamente para fines pedagógicos; sin embargo es claro que su aplicación aún es una meta por lograr. Lo anterior porque existe un desconocimiento en cuanto a la utilidad de las tecnologías y su relación con los contenidos curriculares, en cuanto a la adaptación dentro de la didáctica de la clase.

El desconocimiento produce que exista una falta de usabilidad la cual se entiende como "el grado en el cual un producto puede ser usado por unos usuarios específicos para alcanzar ciertas metas especificadas con efectividad, eficiencia y satisfacción en un contexto de uso especificado" (Cobo, 2005, p.125).

Principalmente para las escuelas unidocentes, el uso de las TIC es un reto, ya que estas poseen dentro de una misma aula estudiantes con diversidad de edades, conocimientos e intereses. Los estudiantes deben compartir el mismo espacio físico y los temas curriculares deben ser correlacionados según el nivel de cada estudiante, haciendo que todos los grados vean el mismo tema, pero con diferente dificultad.

El tipo de escuelas representan $49 \%$ de los centros educativos del país y se encuentran principalmente en zonas alejadas, cerca de la playa, de volcanes o internadas en la montaña (Chaves, 2011, p. 43). Además el contexto socioeconómico generalmente presenta familias con escasez de recursos, dedicados principalmente a la agricultura y ganadería. Las posibilidades de crecimiento son pocas, por ende, la educación debe contemplarse como el mecanismo para romper las brechas.

Los centros educativos unidocentes están gestionados por una única persona, contratada bajo el puesto de profesor de enseñanza unidocente. Este profesional además de realizar funciones pedagógicas, debe de administrar el centro educativo, lo que genera una carga laboral significativa. La administración de este tipo de escuela es exactamente la misma que una escuela regular, esto genera que la organización del tiempo deba ser eficiente, ya que poseen una hora y veinticinco minutos (1:25min) para realizar las gestiones administrativas y la planificación pedagógica.

Según los lineamientos de horarios del Ministerio de Educación (2016), el horario de 1:15 p.m. a 2:40 p.m. debe de ser utilizado para elaborar el planeamiento didáctico, la elaboración de materiales y el cumplimiento de labores relacionadas con la administración y funcionamiento del centro educativo. Lo anterior quiere decir que en este espacio, además de la planificación pedagógica, también debe 
programar reuniones con el patronato escolar, preparar documentos para la supervisión, elaborar cuadros estadísticos e informes, entre otros.

La doble función mencionada genera en los unidocentes un impacto en su salud mental que se traduce en estrés, depresión y ansiedad. Este tipo de enfermedades psicosomáticas alteran el estado anímico y correlacionalmente la motivación, provocando así que la calidad de la educación se vea alterada (Palomares, 1996).

Por tanto, se plantea que las tecnologías de información y comunicación podría ser un insumo al quehacer profesional de los unidocentes. Por ende, el objetivo de la investigación es conocer los aportes de las TIC a la gestión administrativa de las escuelas unidocentes seleccionadas.

\section{Metodología}

Para cumplir con el objetivo de investigación se utilizó un enfoque cualitativo, el cual según Taylor (1987) permite obtener datos descriptivos: recolectando datos hablados o escritos, con las propias palabras de los sujetos y su conducta observable. El enfoque admite la percepción de los sujetos y muestra la realidad subjetiva del contexto en estudio.

\section{Fuentes de información}

Las instituciones en estudio son: Carrera Buena, Carolina Mirambell, Barrio el Carmen y El Socorro, ubicadas en el Circuito 03 del cantón de San Ramón. Carrera Buena cuenta con una matrícula de cinco estudiantes, al igual que la escuela El Socorro. Por su parte Carolina Mirambell y El Socorro tienen matriculados a 17 estudiantes.

Los sujetos informantes fueron cuatro profesores unidocentes y dos asesores de Informática Educativa, un total de cinco sujetos, quienes se seleccionaron por conveniencia y se trató de que pertenecieran a un mismo distrito; lo cual implica que todos pertenecen al Programa de la Fundación Omar Dengo (Multigrado), presentan una cantidad de estudiantes inferior a 20 estudiantes y comparten las mismas condiciones socioeconómicas. Lo anterior con el propósito de que los resultados no variaran en gran medida por el contexto sociocultural de cada escuela,

\section{INSTRUMENTOS}

La investigación se sirve de dos técnicas cualitativas para la recolección de datos. En primer lugar, la entrevista semiestructurada, la cual permite comprender la percepción de los sujetos, además de estar anuente al surgimiento de nuevas variables recolectadas. La entrevista se aplicó a unidocentes del Circuito 03 de San Ramón, específicamente en cuatro escuelas.

La segunda técnica utilizada es la observación no participante, la cual se realizó mediante un proceso sistemático para recolectar la información. Mediante una lista de cotejo, se recopilaron datos observables, esto mientras los unidocentes atendían sus labores administrativas, es decir de 1:20 p.m. a 2:40 p.m. 


\section{RESULTADOS}

A continuación se presentan los resultados con base en las unidades de análisis: conceptualización de escuelas unidocentes, frecuencia y uso de las tic en los centros educativos unidocentes, gestión administrativa en los centros educativos unidocentes y aplicación de las tecnologías de información y comunicación en la gestión administrativa de las escuelas seleccionadas.

\section{Conceptualización de escuelas unidocentes}

Las escuelas unidocentes presentan particularidades muy propias del contexto social donde estén ubicadas. La mayoría, y así es el caso de las escuelas en estudio, tienen una matrícula inferior a 20 estudiantes, se ubican en zonas alejadas y son zonas de bajos recursos.

En el área pedagógica se manifiestan diferencias muy marcadas con respecto a escuelas de otras modalidades; sin embargo a nivel administrativo se comparten prácticamente las mismas funciones que otro tipo de instituciones, siendo el unidocente el encargado del manejo de la escuela a nivel pedagógico y administrativo.

Los unidocentes entrevistados manifiestan que parte importante de trabajar en este tipo de escuelas es la heterogeneidad que existe dentro de una misma aula, con estudiantes que van desde primer grado hasta el sexto, con edades aproximadas entre los 6 y 13 años. El trabajo con estos grupos debe de ser correlacionado, generando una planificación detallada de cada contenido curricular y que el nivel de dificultad varíe dependiendo del nivel

[...] uno tiene que preparar cada clase para cada grado diferente, hacer trabajos cotidianos, extra clases, exámenes y proyectos, todo esto para cada una de las materias. (Docente entrevistado, 2017)

En cuanto al área administrativa, tienen que contar con comités y Junta de Educación, realizar reuniones periódicas con estos y llevar actas de todo lo que se comunica. Deben de elaborar y enviar toda la papelería que exige el Ministerio de Educación, así como dar apoyo a otras instituciones que requieran de información sobre la institución, por ejemplo, el IMAS. Parte del proceso es generar todo tipo de informes y evidenciar cada actividad con fotografías y videos.

Manejar las dos áreas (pedagógica y administrativa) evidentemente requiere de una organización y planificación eficiente; sin embargo, el tiempo estimado para la realización y concertación de las actividades se reduce en un tiempo mínimo, donde el unidocente debe de extender su horario o bien llevar trabajo para el hogar.

Debido a esta cantidad de funciones, los unidocentes entrevistados han manifestado que se han tenido que incapacitar al menos una vez en el último año, debido a casos de estrés, depresión o ansiedad

[...] no pude recibir una capacitación sobre las TIC, porque tuve que incapacitarme por depresión, ya no podía seguir. (Docente entrevistado, 2017)

El estrés al que son sometidos en esta modalidad de escuela se manifiesta en los casos de estrés presentes en los sujetos de estudio. El estrés se intensifica tanto que como consecuencia se manifiesta en depresión, ansiedad e inclusive ambas. Lo anterior altera significativamente el estado anímico, repercutiendo en la motivación. Según la teoría motivacional de Maslow (1943) es necesario primeramente alcanzar las necesidades prioritarias de amor, seguridad y fisiológicas, si estas no fueran alcanzadas no 
se podría encontrar la motivación para otras necesidades como la autorrealización. A medida que se cubren las necesidades mencionadas, la salud mental logra la plenitud humanística (para ampliar el tema ver Brenes, 2018, p.82).

Lo anterior refleja que si no existe una motivación plena, se afecta la autorrealización, afecta directamente el rendimiento del unidocente, generando cansancio, aturdimiento y apatía. Esto no quiere decir que sea falta de profesionalismo, si no que existe una correlación entre motivación y autorrealización, lo cual perjudicaría la dinámica de las clases, la planificación y la calidad de la misma.

Debido a las consecuencias mencionadas anteriormente, es vital comprender la necesidad de que la modalidad unidocente cuente con facilidades para el desarrollo de su labor, principalmente en el área administrativa que es donde se manifiesta más agotamiento. Para esto, se visualiza a las tecnologías de información y comunicación como un aliado que facilita las funciones administrativas y genera un impacto positivo.

\section{Frecuencia y uso de las TIC en los centros educativos unidocente}

Por "frecuencia" se entiende el número de veces que se emplea las tecnologías de información y comunicación para apoyar las labores que demanda el puesto de unidocente; a su vez "uso" se define como las diferentes formas en que las TIC están siendo aplicadas.

Los resultados arrojan que la utilización de la tecnología depende de las funciones que correspondan; es decir, si al unidocente se les exige enviar un documento, se utiliza el recurso de la computadora para remitirlo.

Se manifiesta que la utilización de las TIC ha facilitado algunas labores, ahorrando el tiempo en traslados y mejorando la comunicación. Lo que anteriormente generaba gastos en recursos como fotocopias y pasajes en trasporte, ahora se pueden realizar con el aprovechamiento del correo electrónico.

En el área pedagógica el manejo de las tecnologías de información y comunicación es más constante. Las instituciones al ser favorecidas con el Programa de Informativa Educativa PRONIE-MEP-FOD, cuentan con capacitaciones para el aprovechamiento de los recursos tecnológicos con fines didácticos. Sin embargo, y en concordancia con Paniagua (2016), las tecnologías tienden a ser usado de manera demostrativa, al ser consideradas como recursos de apoyo o de proyección visual. Esta posición concuerda con la teoría que indica que las TIC por sí solas, no presentan un cambio significativo, si no que se requiere de una serie de acciones, en las cuales se incluye el nivel de apropiación del profesorado para que exista un cambio sustancial en su implementación (Castellanos, 2015)

Pese a que cada una de las escuelas está dotada de tecnología, los unidocentes manifiestan que existen limitaciones que provocan que su ejecución se vea disminuida o afectada. Una de ellas es el acceso a Internet. Debido a las características de este tipo de escuelas, la lejanía las excluye de forma parcial de una buena recepción.

Sin Internet no podemos poner a los estudiantes a buscar información y no tengo acceso al correo electrónico. (Docente entrevistado, 2017)

Nótese como existe relación entre los recursos tecnológicos y el acceso a Internet, si presenta malas condiciones limita totalmente el uso de las TIC para las instituciones. Sumado lo anterior al principal factor de uso inadecuado, el desconocimiento del verdadero uso de las tecnologías de información y comunicación. 
Así, el desconocimiento es otras de las limitantes. Los informantes afirman que si ellos conocieran más herramientas y poseyeran competencias tecnológicas tendrían más facilidad para implementar de forma oportuna las TIC; lo cual concuerda con diversos estudios donde se concluye que la formación de maestros en el uso de las TIC en muchos casos no ha sido la adecuada y/o ha adolecido de algunos elementos relevantes. Así mismo, los contenidos han sido utilizados como accesorios a las clases, más no como un apoyo real y base para desarrollar los contenidos (Castellanos, 2015).

\section{Aporte de las tecnologías de información y comunicación aplicado a las funciones del unidocente}

Las funciones de los unidocentes en el área administrativa y pedagógica permiten la utilización de las tecnologías de información y comunicación como un apoyo. Su uso facilita la gestión del centro educativa y evidentemente la didáctica de las clases.

Los unidocentes manifiestan que existe una limitación con el tiempo, ya que el horario administrativo no coincide con todas las necesidades que tienen que cubrir. En este sentido, las TIC han agilizado los tiempos en cuanto a comunicación y traslados principalmente.

Con respecto a los canales de comunicación, los unidocentes expresan que el uso del correo electrónico y el whatasapp han colaborado para un mejor enlace con el asesor de la zona, así como con el Ministerio de Educación; sin embargo se manifiesta que existe un uso desproporcionado de las herramientas.

\section{[...] gastamos horas revisando el correo electrónico y mucha de la información ni siquiera corresponde a casos existentes en la escuela. (Entrevistado, 2017)}

Lo anterior responde a un tema de mala ejecución de las tecnologías de información y comunicación, donde más bien estas han saturado de trabajo a los unidocentes, según indican los informantes. Al respecto, la teoría indica lo siguiente:

Es fundamental resaltar que ninguna herramienta genera impacto por sí sola, todo lo contrario, si es utilizada de forma inadecuada, sin un propósito claro y/o sin involucrar aspectos complementarios, puede incluso menoscabar el propósito inicial de su introducción en cualquier sistema. (Castellanos, 2015, p. 5)

En cuanto al uso de las TIC en la planificación, su utilización como tal es meramente parcial; es decir, únicamente se utiliza el paquete de Office para crear documentos o bien redactar sobre informes previamente realizados. Los entrevistados manifiestan que utilizan alarmas para recordar eventos y utilizan el correo para enviarse planes entre ellos.

En cuanto a la toma de decisiones no existe ningún mecanismo utilizado por los sujetos de información. Ellos expresan que no conocen herramientas que puedan utilizar para ejecutar ese tipo de medidas en el área administrativa y es poco lo que se conoce en general para facilitar la gestión.

Contrario al aporte en cuanto a funciones administrativas, a nivel pedagógico se evidencia que sí hay un acercamiento hacia el verdadero funcionamiento de las TIC, ya que se están utilizando programas y software como complemento de temas curriculares

[...] utilizo la herramienta de Cmaptools para hacer mapas conceptuales y uso mucho Power Point como complemento de algún tema que estemos viendo. (Entrevistado, 2017) 
La contradicción en el uso de la tecnología a nivel administrativo y pedagógico podría asumirse por las capacitaciones que se han brindado a los unidocentes para cambiar la dinámica de la clase. Según mencionan los entrevistados, las capacitaciones para el uso de las TIC en el aula han sido buenas y significativas, y las califican como útiles dependiendo del tema y la facilidad de empleo del estudiante.

Sin embargo, se cree que aún no existe una adecuada usabilidad de las TIC ni a nivel pedagógico ni en el área administrativa. Las TIC como tales no generan un cambio pragmático si estas no son utilizadas de forma diferente a la educación tradicional. Las TIC buscan promocionar el constructivismo, así como las clases dinámicas, donde el estudiante sea participe de su propio conocimiento.

Administrativamente la implementación de las tecnologías es nula, no existe capacitaciones ni autocapacitación por parte de los unidocentes y solo $6 \%$ de quienes trabajan bajo esta modalidad poseen título en Administración Educativa (Chaves, García \& Alvarado, 2010).

Según la UNESCO (2006), las TIC ayudan a agilizar y reducir la información, para poder tener acceso a ella más fácilmente y de manera más eficiente, por ende se mejora a nivel externo e interno la administración educativa; sin embargo, se está confundiendo la forma en que esta debe de ser implementada. El uso de las tecnologías no consiste en tener las mismas prácticas digitales, ni tampoco en utilizar una presentación donde se exponga lo mismo que contiene un texto. Su utilización va más allá, busca crear protagonismo, apropiación y sobre todo generar un impacto positivo en la forma de gestionar administrativamente un centro educativo.

\section{CONCLUSIONES}

Se evidencia que existe un uso de las tecnologías de información y comunicación más pedagógico que administrativo, pues administrativamente se muestra una nula inclusión de los recursos tecnológicos y de emplearse es de forma superficial. En ambas áreas (pedagógica y administrativa) la usabilidad de las TIC no es la más adecuada, ya que se emplean los recursos tecnológicos de la misma forma como se haría manualmente, reproduciendo el mismo modelo tradicional, pero utilizando herramientas digitales.

Se muestra que falta una intención clara sobre la utilización de las TIC, los docentes conocen que es una excelente herramienta, la cual facilita y hace la clase más dinámica, pero no saben cómo aplicarlas.

En el área administrativa, se produce un descontento con las herramientas, ya que consideran que el "abuso" de las TIC les genera más trabajo. Esto podría analizarse como una falta de existencia de educación tecnológica, en cuanto a uso responsable de los canales comunicativos. Por lo anterior, se determina que los aportes de las TIC a la gestión administrativa de estos centros educativos no han sido positivos, y se observan como mecanismos que complican y saturan el trabajo.

Se manifiesta que no existen capacitaciones en cuanto al uso de las TIC a nivel administrativo y esto puede generar que haya un desconocimiento y un temor a su utilización en este campo. En el nivel de gestión educativa, la preparación de los unidocentes deja de lado un respaldo académico como lo es la Administración Educativa, ya que dentro del perfil para laborar no se exige poseer este título universitario.

Se determina que debe de existir un modelo de aplicación de las TIC enfocado en los centros educativos unidocentes, ya que la gran cantidad de funciones que ejercen los educadores provocan la aparición de enfermedades psicosomáticas que influyen negativamente en la calidad de la educación. El modelo debe de comprender las necesidades de las escuelas, de los unidocentes y de los estudiantes, así como su contexto social. A su vez se debe considerar el limitado tiempo de estos profesionales para realizar sus labores. 


\section{REFERENCIAS}

Chaves, L., García, J. \& Alvarado R. (2010). Las escuelas unidocentes en Costa Rica: fortalezas y limitaciones. San José: Universidad de Costa Rica.

Cobo, C. (2005). Organización de la información y su impacto en la usabilidad de las tecnologías interactivas. Tesis doctoral de la Universidad Autónoma de Barcelona. En red. Recuperado de http://www. tdx.cat/bitstream/handle/10803/4090/ccr1de1.pdf?sequence=1

Ministerio de Educación Pública (2016). Lineamientos sobre los horarios para los diferentes ciclos, niveles, ofertas y modalidades del Sistema Educativo costarricense. San José: MEP.

Palomares, A. (1996). El estrés en la educación. Revista de la Facultad de Investigación de Albacete. España. p.153-166.

Fundación Omar Dengo. (2006). Educación y tecnologías digitales. Cómo valorar su impacto social y sus contribuciones a la equidad. San José: FOD.

Paniagua, A. (2016). Uso de las tecnologías de información y comunicación como estrategias de innovación eficientes en el proceso de enseñanza y aprendizaje implementadas por el personal docente de cada área curricular del IV ciclo del Liceo San Roque, Circuito 06 de la Dirección Regional de Educación de Alajuela, durante el segundo semestre del curso lectivo 2016. (Tesis de grado).San José: Universidad Estatal a Distancia.

Castellanos, M. (2015). ¿Son las TIC realmente una herramienta valiosa para fomentar la calidad de la educación? París: UNESCO.

UNESCO. (2009). Medición de las Tecnologías de la Información y la Comunicación (TIC) en Educación. Manual del Usuario ISBN 978-92-9189-092-7, Ref: IEU/TD/10-06. 